\title{
DỨBin
}

Technological University Dublin

ARROW@TU Dublin

Articles

School of Mathematics

2011

\section{Holomorphic Liftings from Infınite Dimensional Spaces}

\author{
Sean Dineen \\ University College Dublin, sean.dineen@ucd.ie \\ Milena Venkova \\ Technological University Dublin, milena.venkova@tudublin.ie
}

Follow this and additional works at: https://arrow.tudublin.ie/scschmatart

Part of the Analysis Commons

\section{Recommended Citation}

S. Dineen and M. Venkova, Holomorphic liftings from infinite dimensional spaces, Mathematical Proceedings of the Royal Irish Academy 111A (2011), 57-68; doi:10.3318/PRIA.2011.111.1.7.

This Article is brought to you for free and open access by the School of Mathematics at ARROW@TU Dublin. It has been accepted for inclusion in Articles by an authorized administrator of ARROW@TU Dublin. For more information, please contact arrow.admin@tudublin.ie, aisling.coyne@tudublin.ie,gerard.connolly@tudublin.ie. Funder: SFI grant R9317

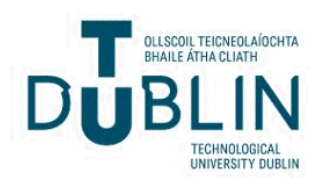




\title{
Holomorphic Liftings from Infinite Dimensional Spaces
}

\author{
Seán Dineen, Milena Venkova*
}

\section{Summary}

We obtain a number of positive solutions to the following holomorphic lifting problem: if $\Omega$ is a domain in a locally convex space $E, X$ and $Y$ are Banach spaces with $Y \subset X$ and quotient mapping $\pi: X \longrightarrow X / Y$, and $f: \Omega \longrightarrow X / Y$ is holomorphic does there exist $h: \Omega \longrightarrow X$ such that $f=\pi \circ h$ ?

\section{Introduction}

Diverse results on the holomorphic lifting problem have been obtained since 1980. In 1980 Aron [1] proved lifting theorems for entire holomorphic functions of compact type. R. Ryan showed, using monomial expansions, in [23] that all holomorphic mappings of bounded type from $\ell_{1}(\Gamma), \Gamma$ an arbitrary indexing set, to $X / Y$ could be lifted to a holomorphic function of bounded type from $\ell_{1}(\Gamma)$ to $X$ and in [2] the authors construct, for every pair of Banach spaces $X$ and $Y$, a universal space $M_{X, Y}$, such that all $Y$-valued holomorphic functions of bounded type defined on $X$ and all $Y$-valued holomorphic functions defined on a balanced domain in $X$ could be lifted holomorphically to $M_{X, Y}$. While investigating the dependence of solutions of operator

*This work was carried out with the partial support of SFI grant R9317. AMS clasification 46G20. Key words: Holomorphic liftings, sheaf cohomology, approximation property, tensor product. 
equations on a holomorphic parameter in [8] we obtained a positive solution to the holomorphic lifting problem for arbitrary Banach spaces when $\Omega$ is a pseudo-convex domain in $\ell_{1}$.

From the linear theory we know that if, for all pairs of Banach spaces $X$ and $Y, Y \subset X$, all $X / Y$-valued linear mappings defined on a Banach space $W$ can be lifted to linear (or polynomial or holomorphic) mappings from $W$ to $X$ then $W$ is isomorphic to $\ell_{1}(\Gamma)$ for some $\Gamma$ and in [9] the authors obtain a similar characterization of $\mathcal{L}_{1}$-spaces using polynomials which are weakly continuous on bounded sets. Thus positive solutions to the holomorphic lifting problem for arbitrary separable $X$ and $Y, Y \subset X$, only occur when $\Omega$ is a pseudo-convex subset of $\ell_{1}$. To obtain further positive results we let $\Omega$ lie in a locally convex space and consider special combinations of Banach spaces. We use a sheaf theory approach in section 2, exactness of the functor $\otimes_{\epsilon}$ in section 3 and in section 4 obtain a number of miscellaneous results.

We let $X, Y, Z$ etc. denote Banach spaces and let $E, F$ etc. denote locally convex spaces, all over the complex numbers $\mathbb{C}$. If $X$ is a Banach space and $\Omega$ is a domain in $E$ let $\mathcal{H}(\Omega, X)$ denote the set of all holomorphic mappings from $\Omega$ to $X$. We let $\mathcal{L}(X, Y)$ denote the set of all continuous linear mappings from $X$ to $Y$ and if $X=Y$ we write $\mathcal{B}(X)$ in place of $\mathcal{L}(X, X)$. We let $E^{\prime}$ denote the space of all continuous linear mappings from $E$ to $\mathbb{C}$ and write $E_{c}^{\prime}$ when this space is endowed with the compact open topology. We refer to $[6,19]$ for background information on infinite dimensional holomorphy and to $[4,24]$ for tensor products.

\section{Short Exact Sequences}

The general holomorphic lifting problem may be presented diagrammatically as follows. If $Y$ is a closed subspace of a Banach space $X, \pi: X \mapsto X / Y$ is the quotient mapping, $\Omega$ is a domain in $E$ and $f \in \mathcal{H}(\Omega, X / Y)$ does there exist $g \in \mathcal{H}(\Omega, X)$ such that the following diagram:

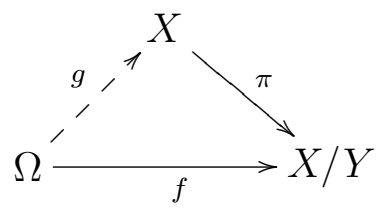

commutes. We find it convenient in this and the following section to use 
short exact sequences. If

$$
0 \longrightarrow Y \stackrel{i}{\longrightarrow} X \stackrel{q}{\longrightarrow} Z \longrightarrow 0
$$

is a short exact sequence of Banach spaces and continuous linear mappings then $i$ is an injective mapping from $Y$ onto the Banach subspace $k e r(q)$ of $X$ and, by the open mapping theorem, we may identify $Y$ with the closed subspace $i(Y)$ of $X$. Furthermore, since $q$ is surjective it is open by the open mapping theorem and we may identify $Z$ with the quotient space $X / Y$. If $Y \simeq i(Y)=\operatorname{ker}(q)$ is a complemented subspace of $X$ we say that the sequence (1) splits. In this case $\operatorname{Image}(q) \simeq Z$ is a complement of $i(Y)$ and, with these identifications, the sequence (1) can be rewritten as

$$
0 \longrightarrow Y \stackrel{i \oplus 0}{\longrightarrow} Y \oplus Z \stackrel{0 \oplus q}{\longrightarrow} Z \longrightarrow 0
$$

If $Y^{\perp}:=\left\{x^{\prime} \in X^{\prime}: x^{\prime}(i(Y))=0\right\}$ denotes the polar of $Y$ in $X^{\prime}$, the strong dual of $X$, we may identify as Banach spaces $Y^{\perp}$ and $Z^{\prime}$ and we obtain the dual short exact sequence

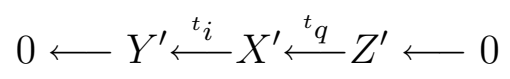

where ${ }^{t} i$ denotes the transpose mapping to $i$ etc. Since the holomorphic lifting problem has, trivially, a positive solution when $Y$ is a complemented subspace of $X$ we will only be interested in the short exact sequences (1) which do not split. However, we shall see later non-trivial examples where (1) does not split but the dual sequence (2) does split.

If $i^{*}(f):=i \circ f$ for all $f \in \mathcal{H}(\Omega, Y)$ and $q^{*}(g):=q \circ g$ for all $g \in \mathcal{H}(\Omega, X)$, where $\Omega$ is a domain in a locally convex space, then it is easily seen that $i^{*}$ is injective and $\operatorname{Im}\left(i^{*}\right)=\operatorname{ker}\left(q^{*}\right)$. This shows that the holomorphic lifting problem has a positive solution for $\Omega$ if and only if the sequence

$$
0 \longrightarrow \mathcal{H}(\Omega, Y) \stackrel{i^{*}}{\longrightarrow} \mathcal{H}(\Omega, X) \stackrel{q^{*}}{\longrightarrow} \mathcal{H}(\Omega, Z) \longrightarrow 0
$$

is exact.

The results we prove depend on various hypotheses on $X, Y, Z, E$ and $\Omega$ and come from different settings. For the reader's convenience we summarise our main results in the following theorem. Unexplained terms will be introduced later. 
Theorem 1 Let

$$
0 \longrightarrow Y \stackrel{i}{\longrightarrow} X \stackrel{q}{\longrightarrow} Z \longrightarrow 0
$$

denote a short exact sequence of Banach spaces. If $\Omega$ is an open subset of the locally convex space $E$ then the sequence

$$
0 \longrightarrow \mathcal{H}(\Omega, Y) \stackrel{i^{*}}{\longrightarrow} \mathcal{H}(\Omega, X) \stackrel{q^{*}}{\longrightarrow} \mathcal{H}(\Omega, Z) \longrightarrow 0
$$

is exact, that is the holomorphic lifting problem has a positive solution, if any of the following conditions hold;

(a) E is a separable Banach space with an unconditional basis, $\Omega$ is pseudoconvex and $Y$ in an $M$-ideal in $X$.

(b) $E$ is a log-convex Fréchet space and $\Omega$ is pseudo-convex,

(c) $E$ is a $\mathcal{D F C}$ space with the approximation property and separable dual and the dual sequence

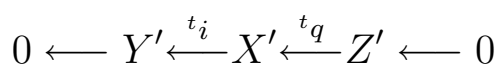

splits.

\section{A Sheaf theory approach to liftings}

For $E$ a fixed locally convex space and $X$ a Banach space we let $\mathcal{O}^{X}$ denote the sheaf of germs of $X$-valued holomorphic functions on $E$. If we are given the short exact sequence of Banach spaces (1) then (3) are the sections over $\Omega$ associated with the sequence of sheaves

$$
0 \longrightarrow \mathcal{O}^{Y} \stackrel{i^{*}}{\longrightarrow} \mathcal{O}^{X} \stackrel{q^{*}}{\longrightarrow} \mathcal{O}^{Z} \longrightarrow 0 .
$$

If (4) is a short exact sequence of sheaves then we obtain the long exact sequence

$$
0 \longrightarrow \mathcal{H}(\Omega, Y) \stackrel{i^{*}}{\longrightarrow} \mathcal{H}(\Omega, X) \stackrel{q^{*}}{\longrightarrow} \mathcal{H}(\Omega, Z) \longrightarrow H^{1}(\Omega, Y) \longrightarrow \cdots
$$


where $H^{1}(\Omega, Y)$ is the first cohomology of $\Omega$ with values in $\mathcal{O}^{Y}$. Thus to show that (3) is exact it suffices to show that (4) is a short exact sequence of sheaves and that $H^{1}(\Omega, Y)=0$. To show that (4) is exact is equivalent to showing the existence of local liftings. We call on results of Lempert $[14,16]$ to obtain the cohomology result we require. This is the method we used to prove Proposition 1 in [8].

A separable Banach space has the bounded approximation property if and only if there exists a sequence $\left(T_{m}\right)_{m=1}^{\infty} \subset \mathcal{B}(X)$ such that $T_{m}(x) \longrightarrow x$ for all $x \in X$. A separable Banach space has the bounded approximation property if and only if it is isomorphic to a complemented subspace of a Banach space with a Schauder basis. In particular, we see that the bounded approximation property is inherited by complemented subspaces of separable Banach spaces with the bounded approximation property. By the Uniform Boundedness Principle, $\lambda:=\sup _{m}\left\|T_{m}\right\|<\infty$ and we say that $X$ has the $\lambda$-approximation property.

The following lemma shows how linear liftings (respectively linear liftings with bounds) can be extended to obtain polynomial liftings (respectively local holomorphic liftings). Proposition 2.3 and the remarks prior to it in [10] give counterexamples to the lifting problem when any of the hypothesis in Proposition 2 are removed.

Lemma 1 If the Banach space $X$ has the $\lambda$-approximation property then $\widehat{\otimes}_{n, s, \pi} X$, the $n$-fold completed symmetric projective tensor product of $X$ with itself $n$ times, has the $\lambda^{n}$-approximation property.

Proof. If $\left(T_{m}\right)_{m=1}^{\infty}$ denotes the sequence defining the $\lambda$-approximation property then $T_{m}^{n}:=T_{m} \otimes \cdots \otimes T_{m}$ (n-times) $\in \mathcal{B}\left(\widehat{\otimes}_{\pi, s, n} X\right)$ and $\left\|T_{m}^{n}\right\| \leq$ $\left\|T_{m}\right\|^{n} \leq \lambda^{n}$ for all $m$ and $n$. If $x \in X$, then

$$
\begin{aligned}
\left\|T_{m}^{n}\left(\otimes_{n} x\right)-\otimes_{n} x\right\| & \leq\left\|\sum_{j=1}^{n}\left(T_{m}^{j-1}\left(\otimes_{j-1} x\right)\right) \otimes\left(T_{m}(x)-x\right) \otimes_{n-j} x\right\| \\
& \leq(n+1) \sup _{1 \leq j \leq n}\left\{\left\|T_{m}\right\|^{j-1} \cdot\left\|T_{m}(x)-x\right\| \cdot\|x\|^{n-j}\right\} .
\end{aligned}
$$

Linearity, density, and boundedness of the sequence $\left(\left\|T_{m}\right\|\right)_{m=1}^{\infty}$ imply that $T_{m}^{n}(\theta) \longrightarrow \theta$ for all $\theta \in \widehat{\otimes}_{n, s, \pi} X$ as $m \longrightarrow \infty$. This completes the proof. 
A closed subspace $Y$ of a Banach space $X$ is called an M-ideal if there exists a projection $P$ from $X^{\prime}$ into $X^{\prime}$ with range $Y^{\perp}$ such that

$$
\|\phi\|=\|P(\phi)\|+\|\phi-P(\phi)\|
$$

for all $\phi \in X^{\prime}$. Any closed ideal in a $\mathcal{C}^{*}$ algebra is an $M$-ideal, in particular the Banach space $c_{0}$ is an M-ideal in $\ell_{\infty}$, and $\mathcal{K}(H)$, the compact linear operators from a Hilbert space into itself, is an M-ideal in $\mathcal{B}(H)$. If $1<p \leq q<\infty$, then $\mathcal{K}\left(\ell_{p}, \ell_{q}\right)$, the compact linear operators from $\ell_{p}$ to $\ell_{q}$, is an M-ideal in $\mathcal{L}\left(\ell_{p}, \ell_{q}\right)$ (by [21] if $X$ and $Y$ are Banach spaces and $Y$ has an unconditional basis then either $\mathcal{K}(X, Y)=\mathcal{L}(X, Y)$ or $\mathcal{K}(X, Y)$ is an uncomplemented subspace of $\mathcal{L}(X, Y))$. We refer to [10] for further information on M-ideals.

The following proposition includes part (a) of Theorem 1.

Proposition 1 If $W$ is a separable Banach space with the $\lambda$-approximation property, $Y$ is an $M$-ideal in the Banach space $X$, and the sequence (1) is exact then the sequence (4) is exact for sheaves of holomorphic germs on $W$. If, in addition, $W$ has an unconditional basis then the sequence (3) is exact for every pseudo-convex open subset $\Omega \in W$.

Proof. To show exactness at the germ level consider a $Z$-valued holomorphic function $f$ on an open ball centered at the origin. Each such function has a Taylor series expansion $\sum_{n=0}^{\infty} P_{n}$ such that $\sum_{n=0}^{\infty} \beta^{n}\left\|P_{n}\right\|<\infty$ for some $\beta>0$. We may identify each $P_{n}$ with the mapping $Q_{n} \in \mathcal{L}\left(\widehat{\otimes}_{n, s, \pi} W ; Z\right)$ where $P_{n}(x)=Q_{n}(x \otimes \cdots \otimes x)$ for all $x \in W$. We then have $\left\|P_{n}\right\|=\left\|Q_{n}\right\|$. Since $\widehat{\otimes}_{n, s, \pi} W$ is easily seen to be separable, a result due to Ando, and, independently, to Choi an Effros (see [10] p. 59) and Lemma 1 imply that there exists $R_{n} \in \mathcal{L}\left(\widehat{\otimes}_{n, s, \pi} W ; X\right)$ such that $q \circ R_{n}=Q_{n}$ and $\left\|R_{n}\right\| \leq \lambda^{n}\left\|Q_{n}\right\|$. If $S_{n}(x)=R_{n}(x \otimes \cdots \otimes x)$ for $x \in W$ then $S_{n}$ is a n-homogeneous polynomial from $W$ to $X, q \circ S_{n}=P_{n}$ and $\left\|S_{n}\right\|=\left\|R_{n}\right\| \leq \lambda^{n}\left\|P_{n}\right\|$. If $0<\epsilon<\beta / \lambda$, then $\sum_{n=0}^{\infty} \epsilon^{n}\left\|S_{n}\right\| \leq \sum_{n=0}^{\infty} \epsilon^{n} \lambda^{n}\left\|P_{n}\right\| \leq \sum_{n=0}^{\infty} \beta^{n}\left\|P_{n}\right\|<\infty$. Hence $g:=\sum_{n=0}^{\infty} S_{n}$ is a holomorphic germ at the origin and, as $\pi \circ g=f$, we have a local lifting and (4) is exact.

If $W$ has an unconditional basis and $\Omega$ is pseudo-convex then $H^{1}(\Omega ; Y)=$ 0 and, as noted above, this implies that (3) is exact. This completes the proof.

By [10], p.59, liftings do not always exist, even for linear mappings if either the $\lambda$-approximation property or separability are not assumed.

Part (b) of Theorem 1 is proved in a very similar way. 
Definition 1 [15] A Fréchet space $E$ is log-convex space if there exists $p: \mathbb{R} \times \mathbb{N} \mapsto(0, \infty)$ such that the following hold:

(a) $\log p(\cdot, n)$ is convex for all $n \in \mathbb{N}$,

(b)

$$
E=\left\{\left(x_{n}\right)_{n=1}^{\infty}:\left\|\left(x_{n}\right)_{n=1}^{\infty}\right\|_{\theta}<\infty\right\} \text { for all in } \mathbb{R}
$$

where

$$
\|x\|_{\theta}:=\sum_{n} p(\theta, n)\left|x_{n}\right|
$$

(c) the topology on $E$ is generated by the semi-norms $(\|\cdot\|)_{\theta \in \mathbb{R}}$.

A log-convex Fréchet space has an absolute basis. The Banach space $\ell_{1}$ is log-convex and a Fréchet nuclear space with Schauder basis is log-convex if and only if it has property $D N$. A result of Vogt [?] says that a Fréchet nuclear space has $D N$ if and only if it is linearly isomorphic to a subspace of $s$ (the space of rapidly decreasing sequences). If $\mathbb{D}$ is the open unit disc in $\mathbb{C}$ then the space $\left(\mathcal{H}(\mathbb{D}), \tau_{0}\right)$ is not log-convex.

Proposition 2 If $E$ is a log-convex Fréchet space, $\Omega$ is a pseudo-convex open subset of $E$ and (1) is exact, then (2) is exact.

Proof. Since log-convex Fréchet spaces are projective limits of $\ell_{1}$ spaces, the existence of local liftings follows from either Lemma 1 or the use of monomial expansions as in [23] or Proposition 7 below. Hence the sequence (2) is exact. By [16] the cohomology group $H^{1}(\Omega ; Y)=0$ and hence we may apply (5) to complete the proof.

\section{Exactness of the $\otimes_{\epsilon}$ functor}

Our approach in this section is to establish exactness of the sequence

$$
0 \longrightarrow \mathcal{H}(\Omega) \widehat{\otimes}_{\epsilon} Y \stackrel{i_{\Omega}}{\longrightarrow} \mathcal{H}(\Omega) \widehat{\otimes}_{\epsilon} X \stackrel{q_{\Omega}}{\longrightarrow} \mathcal{H}(\Omega) \widehat{\otimes}_{\epsilon} Z \longrightarrow 0
$$

in place of $(3)$, where $i_{\Omega}:=I_{\mathcal{H}(\Omega)} \otimes i$ and $q_{\Omega}:=I_{\mathcal{H}(\Omega)} \otimes q$, and afterwards to examine the relationship between $\mathcal{H}(\Omega, X)$ and $\mathcal{H}(\Omega) \widehat{\otimes}_{\epsilon} X$. In this case we are assuming that $\mathcal{H}(\Omega)$ is endowed with a locally convex space structure.

The following definition and proposition are given in [11]. 
Definition 2 A short exact sequence of Banach spaces

$$
0 \longrightarrow Y \stackrel{i}{\longrightarrow} X \stackrel{q}{\longrightarrow} Z \longrightarrow 0
$$

is an $(\epsilon L)$-triple if for every Banach space $W$ the sequence

$$
0 \longrightarrow W \widehat{\otimes}_{\epsilon} Y \longrightarrow W \widehat{\otimes}_{\epsilon} X \longrightarrow W \widehat{\otimes}_{\epsilon} Z \longrightarrow 0
$$

is exact.

Proposition 3 (a) The short exact sequence of Banach spaces

$$
0 \longrightarrow Y \stackrel{i}{\longrightarrow} X \stackrel{q}{\longrightarrow} Z \longrightarrow 0
$$

is an $(\epsilon L)$-triple if and only if the dual short exact sequence splits.

(b) If

$$
0 \longrightarrow Y \stackrel{i}{\longrightarrow} X \stackrel{q}{\longrightarrow} Z \longrightarrow 0
$$

is an $(\epsilon L)$-triple then the sequence

$$
0 \longrightarrow E \widehat{\otimes}_{\epsilon} Y \stackrel{i_{E}}{\longrightarrow} E \widehat{\otimes}_{\epsilon} X \stackrel{q_{E}}{\longrightarrow} E \widehat{\otimes}_{\epsilon} Z \longrightarrow 0
$$

is exact for any Fréchet space E.

\section{Example.}

1. If $J_{X}$ denotes the canonical mapping from a Banach space $X$ into its bidual $X^{\prime \prime}$ then we have the direct sum decomposition $X^{\prime \prime \prime}=\left(J_{X} X\right)^{\perp} \oplus$ $J_{X^{\prime}}\left(X^{\prime}\right)$ and hence the short exact sequence

$$
0 \longrightarrow X \stackrel{i}{\longrightarrow} X^{\prime \prime} \stackrel{q}{\longrightarrow} X^{\prime \prime} / X \longrightarrow 0
$$

is an $(\epsilon L)$-triple.

2. $[11,13]$ Let $X$ an $Y$ be Banach spaces and suppose either $X^{\prime}$ or $Y$ has the bounded approximation property. Let $\mathcal{K}(X ; Y)$ denote the space of compact linear mappings from $X$ to $Y$ and let $\mathcal{L}(X ; Y)$ denote the space of continuous linear mappings from $X$ to $Y$. Then

$$
0 \longrightarrow \mathcal{K}(X ; Y) \stackrel{i}{\longrightarrow} \mathcal{L}(X ; Y) \stackrel{q}{\longrightarrow} \mathcal{L}(X ; Y) / \mathcal{K}(X ; Y) \longrightarrow 0
$$

is an $(\epsilon L)$-triple. 
3. [12] If either $Y$ is a $\mathcal{L}_{\infty}$ space, $X$ is a $\mathcal{L}_{2}$ space or $Z$ is a $\mathcal{L}_{1}$ space then

$$
0 \longrightarrow Y \stackrel{i}{\longrightarrow} X \stackrel{q}{\longrightarrow} Z \longrightarrow 0
$$

is an $(\epsilon L)$ triple.

For our next result we need to recall a definition and some known results. If $E$ and $F$ are locally convex spaces then $E \epsilon F:=\mathcal{L}_{\epsilon}\left(E_{c}^{\prime} ; F\right)$ is the space of all continuous linear mappings from $E_{c}^{\prime}$ to $F$ endowed with the topology of uniform convergence on the equicontinuous subsets of $E^{\prime}$. A topological space is a $k$ space if continuity on compact sets implies continuity. If $F$ is a Fréchet space and $E=F_{c}^{\prime}$ we call $E$ a $\mathcal{D} \mathcal{F C}$ space.

Proposition 4 (a) ([3, 22]) If $E$ is a complete locally convex space with the approximation property then $E \widehat{\otimes}_{\epsilon} F=E \epsilon F$.

(b) ([22] Corollary 2.8.) If $\Omega$, an open subset of the locally convex space $E$, is a $k$ space and $F$ is a complete locally convex space then $\left(\mathcal{H}(\Omega, F), \tau_{0}\right)=$ $\left(\mathcal{H}(\Omega), \tau_{0}\right) \epsilon F$.

(c) ([18] Theorems 7.4 and 7.6.) If $\Omega$ is an open subset of a $\mathcal{D F C}$ space $E$ then $\Omega$ is a $k$ space and if $E_{c}^{\prime}$ is separable then $\left(\mathcal{H}(\Omega), \tau_{0}\right)$ is a Fréchet space.

(d) ([7] Theorem 4.3.) If $\Omega$ is an open subset of the $\mathcal{D} \mathcal{F C}$ with the approximation property then $\left(\mathcal{H}(\Omega), \tau_{0}\right)$ has the approximation property.

Our next result proves part (c) of Theorem 1.

Proposition 5 If $E$ is a $\mathcal{D} \mathcal{F C}$ space with the approximation property, $E_{c}^{\prime}$ is separable, $\Omega$ is an open subset of $E$ and

$$
0 \longrightarrow Y \stackrel{i}{\longrightarrow} X \stackrel{q}{\longrightarrow} Z \longrightarrow 0
$$

is an $(\epsilon L)$-triple then

$$
0 \longrightarrow \mathcal{H}(\Omega: Y) \stackrel{i^{*}}{\longrightarrow} \mathcal{H}(\Omega: X) \stackrel{q^{*}}{\longrightarrow} \mathcal{H}(\Omega: Z) \longrightarrow 0
$$

is exact. 
Proof. By Proposition $4(\mathrm{~d}),\left(\mathcal{H}(\Omega), \tau_{0}\right)$ has the approximation property, and hence by Proposition 4(a), (b) and (c),

$$
\left(\mathcal{H}(\Omega), \tau_{0}\right) \widehat{\otimes}_{\epsilon} W=\left(\mathcal{H}(\Omega), \tau_{0}\right) \epsilon W=\left(\mathcal{H}(\Omega ; W), \tau_{0}\right)
$$

for any Banach space $W$. By Proposition $4(\mathrm{c}),\left(\mathcal{H}(\Omega), \tau_{0}\right)$ is a Fréchet space and it suffices to apply Proposition 3(b) to complete the proof.

By Theorem 7.2 in [18] the proof in Proposition 5 extends to give the same result for entire functions on an arbitrary $\mathcal{D} \mathcal{F C}$ space.

\section{$5 \quad$ Miscellaneous results}

A number of results from infinite dimensional holomorphy can be combined with the results of the previous sections to extend the range of positive solutions to the holomorphic lifting property and, in some cases, to provide alternative proofs of special cases of results already proved. We confine ourselves to a few examples.

Proposition 6 Let $E$ denote a locally convex space which is a projective limit of locally convex spaces $\left(E_{\alpha}, \pi_{\alpha}\right)_{\alpha \in \Gamma}$ where $\Gamma$ is a directed set and each $\pi_{\alpha}$ is a surjective open mapping. Let $Y$ denote a closed subspace of the Banach space $X$ with quotient mapping $q: X \longrightarrow X / Y$. If for each $\alpha \in \Gamma$, each pseudo-convex open set $U_{\alpha} \subset E_{\alpha}$ and each $f \in \mathcal{H}\left(U_{\alpha}, X / Y\right)$ there exists $g \in \mathcal{H}\left(U_{\alpha}, X\right)$ such that $f=q \circ g$ then for all $U$ open pseudo-convex in $E$ and all $f \in \mathcal{H}(U, X / Y)$ there exists $g \in \mathcal{H}(U, X)$ such that $f=q \circ g$.

Proof. If $\Omega$ is a pseudo-convex open subset of $E, f \in \mathcal{H}(\Omega, X / Y)$, then by [6], p.387, there is an $\alpha \in \Gamma$ and $f_{\alpha}: \pi_{\alpha}(\Omega) \longrightarrow X / Y$ such that $\pi_{\alpha}(\Omega)$ is a pseudo-convex open subset of $E_{\alpha}, \Omega=\pi_{\alpha}^{-1}\left(\pi_{\alpha}(\Omega)\right), f_{\alpha} \in \mathcal{H}\left(\pi_{\alpha}(U), X / Y\right)$ and $f=f_{\alpha} \circ \pi_{\alpha}$. By our hypothesis there exists $g_{\alpha} \in \mathcal{H}\left(\pi_{\alpha}(\Omega), X\right)$ such that $f_{\alpha}=q \circ g_{\alpha}$. Hence $f=q \circ g_{\alpha} \circ \pi_{\alpha}$. This completes the proof.

If, in the previous lemma, $E$ is a Fréchet space and each $E_{\alpha}$ is a Banach space then $E$ is called a quojection. The space $\mathbb{C}^{\mathbb{N}}$ is a quojection and thus we obtain a positive solution to the holomorphic lifting problem for any pseudo-convex open subset of $\mathbb{C}^{\mathbb{N}}$ and any quotient mapping of Banach spaces. Since $H^{1}\left(\mathbb{C}^{\mathbb{N}}, X\right) \neq 0$ for any Banach space $X[7,16,17]$ this shows 
that vanishing cohomology for pseudo-convex domains, as in Proposition 1, is not necessary in order to obtain holomorphic liftings. $\mathbb{C}^{N} \times \mathbb{C}^{(N)}$ is another example in which the hypothesis of Lemma 2 are satisfied since $\mathbb{C}^{N} \times \mathbb{C}^{(N)}$ is the projective limit of $\mathbb{C}^{n} \times \mathbb{C}^{(N)} \approx \mathbb{C}^{(N)}$ and Proposition 5 applies to the $\mathcal{D F C}$ space $\mathbb{C}^{(N)}$.

A locally convex space $E$ which is reflexive, nuclear and dual nuclear and which has a (Schauder) basis is called a fully nuclear space with basis. Fréchet nuclear spaces with basis, their strong duals, and countable products and sums of fully nuclear spaces with basis are fully nuclear spaces with basis. The spaces of test functions $\mathcal{D}\left(\mathbb{R}^{n}\right)$ and $\mathcal{E}\left(\mathbb{R}^{n}\right)$, with their usual locally convex topologies, and their strong duals, the spaces of distributions $\mathcal{D}^{\prime}\left(\mathbb{R}^{n}\right)$ and $\mathcal{E}^{\prime}\left(\mathbb{R}^{n}\right)$, and $\mathbb{C}^{N} \times \mathbb{C}^{(N)}$ are fully nuclear with a basis. If $\Omega$ is an open polydisc in a fully nuclear space with basis then the monomials form an absolute basis for $\mathcal{H}(\Omega)$ with respect to any of the usual topologies (see [6]). Moreover, if $X$ is a Banach space and $\left(a_{m}\right)_{m \in N^{(N)}} \subset X$ then $\sum_{m \in N^{N}} a_{m} z^{m} \in \mathcal{H}(\Omega, X)$ if and only if for every $K$ compact in $\Omega$ there exists $V$ open, $K \subset V \subset$ $\Omega$, such that $\sum_{m \in N^{(N)}}\left\|a_{m}\right\| \cdot\left\|z^{m}\right\|_{V}<\infty$. The method of proof used in the following proposition was used by Ryan in [23] to lift entire functions of bounded type on $\ell_{1}$. The basis hypothesis in the following proposition can be removed in certain cases for entire functions by using holomorphic functions of nuclear type as in [8]. Moreover, the method of proof can be adapted in a straightforward manner to obtain lifting results for other classes of holomorphic functions on fully nuclear spaces with basis, e.g. holomorphic functions which are bounded on compact sets (the so called hypo-analytic functions).

Proposition 7 If $Y$ is a subspace of the Banach space $X, \pi: X \longrightarrow$ $X / Y$ is the quotient mapping, $\Omega$ is an open polydisc in the fully nuclear space with basis $E$ and $f \in \mathcal{H}(\Omega, X / Y)$ then there exists $g \in \mathcal{H}(\Omega, X)$ such that $\pi \circ g=f$.

Proof. Let $f(z):=\sum_{m \in N^{N}} a_{m} z^{m} \in \mathcal{H}(\Omega, X / Y)$. For each $m \in N^{(N)}$ choose $b_{m} \in X$ such that $\pi\left(b_{m}\right)=a_{m}$ and $\left\|b_{m}\right\| \leq 2\left\|a_{m}\right\|$. The function $g(z):=\sum_{m \in N^{N}} b_{m} z^{m} \in \mathcal{H}(\Omega, X)$ is the required holomorphic lifting.

The same approach can be used to obtain variations of the result in Proposition 5. The removal of conditions on the range spaces $X$ and $X / Y$ in Proposition 5 is replaced in Proposition 8 by additional assumptions on 
the domain space. A locally convex space in which each closed bounded set in compact is called Montel and the strong dual of a Fréchet Montel space is called a $\mathcal{D} \mathcal{F} \mathcal{M}$ space. An entire mapping between Banach spaces is of bounded type if it is bounded on bounded sets.

Proposition 8 If the $\mathcal{D} \mathcal{F} \mathcal{M}$ space $E$ has an absolute basis, if $Y$ is a subspace of the Banach space $X, \pi: X \longrightarrow X / Y$ is the quotient mapping, and $f \in \mathcal{H}(E, X / Y)$ then there exists $g \in \mathcal{H}(E, X)$ such that $\pi \circ g=f$.

Proof. By Example 3.11 in [6] (see also $[18,20]$ ) there exists a mapping $\phi: E \longrightarrow \ell_{1}$ and a holomorphic mapping of bounded type $h_{1} \in \mathcal{H}\left(\ell_{1}, X / Y\right)$ such that $f=h_{1} \circ \phi$. The result of Ryan, [23], quoted in the introduction, shows that there exists $h_{2} \in \mathcal{H}\left(\ell_{1}, X\right)$ such that $h_{1}=\pi \circ h_{2}$. The mapping $g:=h_{2} \circ \phi$ is the required holomorphic lifting.

\section{Fredholm Operators}

In this section we apply the lifting results from earlier sections to Fredholm operators on a Banach space. This was one of our original motivations for considering lifting problems. In a further paper on generalised inverses we obtain extensions of Proposition 9 below by taking a different approach. We let $\Phi(X)$ denote the space of Fredholm mappings from the Banach space $X$ into itself. The following proposition extends Satz 2.3 in [12].

Proposition 9 Let $\Omega$ denote a domain in a locally convex space $E$ and let $X$ denote a Banach space and suppose that either of the following conditions are satisfied:

(1) $E$ is a Banach space with an unconditional basis, $\Omega$ is pseudo-convex, and $\mathcal{K}(X)$ is an $M$-ideal in $\mathcal{L}(X)$,

(2) $E$ is a $\mathcal{D} \mathcal{F C}$ space with separable dual and the approximation property, and $X$ has the bounded approximation property

If $f \in \mathcal{H}(\Omega ; \Phi(X))$ then there exists $g \in \mathcal{H}(\Omega ; \mathcal{L}(X))$ such that $f(z) g(z)-$ $I_{X} \in \mathcal{K}(X)$ and $g(z) f(z)-I_{X} \in \mathcal{K}(X)$ for all $z \in \Omega$. 
Proof. Let $\pi$ denote the quotient homomorphism from the Banach algebra $\mathcal{L}(X)$ into the Calkin algebra $\mathcal{L}(X) / \mathcal{K}(X)$. Since $f(z)$ is Fredholm for all $z \in \Omega, \pi \circ f$ is a holomorphic mapping from $\Omega$ into the invertible elements in a Banach algebra. Hence $g:=(\pi \circ f)^{-1}$ is holomorphic. By Theorem 1 (a) and (c) and our hypothesis there exists $h \in \mathcal{H}(\Omega ; \mathcal{L}(X))$ such that $\pi \circ h=g$. Since

$(\pi \circ(f \cdot h))(z)=\pi(f(z) \cdot h(z))=\pi(f(z)) \cdot \pi(h(z))=(\pi \circ f)(z) \cdot g(z)=I_{\mathcal{L}(X) / \mathcal{K}(X)}$, $f(z) h(z) \in I_{X}+\mathcal{K}(X)$. Similarly $h(z) f(z) \in I_{X}+\mathcal{K}(X)$ and this completes the proof.

Our previous examples show that the hypothesis on $X$ in the above proposition are satisfied by all $\ell_{p}, 1<p<\infty$. The function $g$ in Proposition 9 is called a regulariser for $f$.

\section{References}

[1] R.M. Aron, Extension and Lifting Problems for Analytic Mappings, Functional Analysis: Surveys and Recent Results II, Proceeings of the Paderborn Conference on Functional Analysis 1979, Ed. K-D. Bierstedt and B. Fuchssteiner, North-Holland Mathematical Stuies, 38, 1980, 257-267.

[2] R.M. Aron, L.A. Moraes and O.W. Paques, Lifting of Holomorphic Mappings, Proc. Royal Irish Acad., Sect. A, 94, 1, 1994, 119-126.

[3] K-D. Bierstedt, The approximation-theoretic localization of Schwartz's approximation property for weighted locally convex function spaces and some examples, Functional Analysis, Holomorphy, and Approximation Theory, Proceeings, Rio de Janeiro, 1978, Ed. S. Machado, SpringerVerlag Lecture Notes, 843, 1981, 93-149.

[4] A. Defant, K. Floret, Tensor Norms and Operator Ideals, North-Holland Mathematical Stuies, 1993.

[5] S. Dineen, Cousin's first problem on certain locally convex topological spaces, An. Acad. Brasil. Cienc., 48, 1976, 11-12. 
[6] S. Dineen, Complex Analysis on Infinite Dimensional Spaces, SpringerVerlag Monographs in Mathematics, 1999.

[7] S. Dineen, J. Mujica, The approximation property for spaces of holomorphic functions on infinite dimenional spaces I, J. Approx. Theory, 126, 2004, 141-156.

[8] S. Dineen, I. Patyi, M.Venkova, Inverses Depending Holomorphically on a Parameter in a Banach Space, J. Funct. Anal., 237, 2006, 338-349..

[9] M. González, J.M. Gutiérrez, Extension and Lifting of Polynomials, Arch. Math., 81, 2003, 431-438.

[10] P.Harmand, D.Werner, W.Werner, M-ideals in Banach Spaces and Banach Algebras, Lecture Notes in Mathematics, 1547, Springer-Verlag, 1993.

[11] W. Kaballo, Lifting-Sätze Für Vektorfunctionen Und $(\varepsilon L)$-Räume, J. Reine Angew. Math., 309, 1979, 55-85.

[12] W. Kaballo, D. Vogt, Lifting-Probleme Für Vectorfunktionen ind \&Sequenzen, Manuscripta Math., 32, 1, 1980, 1-27.

[13] W. Kaballo, Lifting Theorems for Vector Valued Functions and the $\epsilon-$ Tensor Product, Functional Analysis: Surveys and Recent Results, Proceeings of the Paderborn Conference on Functional Analysis, Ed. KD. Bierstedt and B. Fuchssteiner, North-Holland Mathematical Studies, 1977, 149-166.

[14] L. Lempert, The Dolbeault complex in infinite dimensions III, Invent. Math., 142, 2000, 579-603.

[15] L. Lempert, On Fréchet Spaces with a Dominant Norm, Math. Proc. Royal Irish Acad., 102A, 2, 2002, 127-129.

[16] L. Lempert, Analytic Cohomology in Fréchet Spaces, Comm. Anal. Geom., 11, 1, 2003, 17-32.

[17] R. Meise, D. Vogt, Counterexamples in holomorphic functions on nuclear Fréchet spaces, Math. Z., 182, 1983, 167-177. 
[18] J.Mujica, Domains of Holomorphy in (DFC)-space, Functional Analysis, Holomorphy and Approximation Theory, Ed. Silvio Machado, Lecture Notes in Math., 843, 1981, 500-533.

[19] J. Mujica, Complex Analysis in Banach Spaces, North-Holland Mathematical Studies, 120, 1986.

[20] L.Nachbin, On pure uniform holomorphy in spaces of holomorphic germs, Results Math., 8, 1985, 117-122.

[21] A.E. Tong, D.R. Wilken, The uncomplemented subspaces of $K(E, F)$. Studia Math., 37, 1970-71, 227-236.

[22] M. Schottenloher, $\epsilon$-Product and Continuation of Analytic Mappings, Analyse fonctionelle et Applications, Ed. L. Nachbin, Hermann, Paris, 1975, 260-275.

[23] R.A. Ryan, Holomorphic functions on $\ell_{1}$, TAMS, 302, 7, 1987, 797-811.

[24] R. A. Ryan, Introduction to Tensor Products of Banach spaces, Springer Monographs in Mthematics, Springer-Verlag, 2002.

[25] D. Vogt, Subspaces and Quotients of (s), Functional Analysis: Surveys and Recent Results, Proceeings of the Paderborn Conference on Functional Analysis, Ed. K-D. Bierstedt and B. Fuchssteiner, North-Holland Mathematical Studies, 1977, 167-187.

Department of Mathematics,

University College Dublin,

Dublin 4,

Ireland. 